\title{
Cardiac Rehabilitation and Exercise Prescription in Symptomatic Patients with Non-Obstructive Coronary Artery Disease-a Systematic Review
}

\author{
Christine K. Kissel, $M D^{1,2, *}$ \\ Dimitra Nikoletou, PhD ${ }^{3}$
}

\author{
Address \\ ${ }^{*}, 1$ Department of Cardiology, University Heart Center, University Hospital Zurich, \\ Zurich, Switzerland \\ Email: Christine.Kissel@usz.ch \\ ${ }^{2}$ Msc Sports Cardiology, Cardiology Clinical Academic Group, St George's, \\ University of London, London, UK \\ ${ }^{3}$ Emergency, Cardiovascular and Critical Care Research Group, Faculty of Health, \\ Social Care and Education (Joint Faculty), Kingston University \& St George's \\ University of London, London, UK
}

Published online: 18 August 2018

(C) The Author(s) 2018

This article is part of the Topical Collection on Sports Cardiology

Keywords Microvascular angina $\cdot$ Coronary artery disease $\cdot$ Exercise $\cdot$ Rehabilitation

\begin{abstract}
Purpose of review Non-obstructive coronary artery disease (NOCAD) on coronary angiography is a common finding in patients with stable angina. Angina in NOCAD patients is thought to be caused by endothelial dysfunction of the epicardial coronary arteries and/or the microvasculature. Treatment is empiric, and $30 \%$ of patients remain symptomatic in spite of therapy. It is well known that physical exercise can improve endothelial function. The goal of this review was to assess the current literature on effects of physical exercise in NOCAD patients with angina. Therefore, a literature search was conducted to March 13, 2018 using the following search terms: syndrome $X$, microvascular angina, non-obstructive coronary artery disease and exercise training, cardiac rehabilitation, endothelial function. All original publications were included which examined the effect of a cardiac rehabilitation (CR) program or exercise training (ET) on patients with angina and NOCAD.
\end{abstract}


Recent findings Eight studies, of which four were randomized controlled studies, examined 218 participants, 162 in an intervention and 56 in control groups. Most patients were women $(97.7 \%)$. Exercise programs varied from 8 weeks to 4 months at moderate intensity and some included relaxation therapy. The studies examined the effect of CR on exercise capacity, quality of life ( $Q \circ \mathrm{L})$, and perfusion defects. CR increased exercise capacity, oxygen uptake, symptom severity, and QoL. Myocardial perfusion improved.

Summary CR appears to be beneficial in symptomatic patients with NOCAD, improving exercise capacity and QoL and reducing severity of symptoms and myocardial perfusion defects. Data is limited to a small number of predominantly female patients. Further larger trials are warranted to determine the optimal rehabilitation protocols and define its long-term benefits.

\section{Introduction}

Non-obstructive coronary artery disease (NOCAD) is often found on coronary angiograms performed in patients with stable angina, with up to $60 \%$ of women having NOCAD $[1 \bullet \bullet$. Angina in the context of NOCAD appears to be caused by endothelial dysfunction at the epicardial and/or microvascular level [2•]. Albeit a heterogonous population, the consensus is that inappropriate coronary vasodilator capacity is the common denominator $[2 \bullet]$. Patients previously diagnosed with "cardiac syndrome X" (CSX) also belong to this entity and are now classified as microvascular angina [3].

Numerous studies indicate an increased risk of cardiovascular events in these patients and high costs due to frequent rehospitalization, repeat diagnostic procedures, and medical therapy $[1 \bullet \bullet, 4,5]$. Approximately one third of patients remain symptomatic in spite of therapeutic interventions, which are often empirical [5,
$6 \bullet]$. NOCAD angina is associated with depression and decreased quality of life (QoL) [7]. The duration and intensity of symptoms are independent predictors of reduced QoL in women with NOCAD [8].

It is well known that exercise improves endothelial function in patients with coronary artery disease (CAD) [9] and heart failure [10]. Furthermore, exercise training (ET) is a fundamental part of cardiac rehabilitation (CR) which has been repeatedly shown to reduce cardiovascular mortality and rehospitalization rates and improve psychological wellbeing and risk factor control in patients with CAD [11].

The goal of the systematic review was to appraise available literature on CR in patients with NOCAD/ microvascular angina and to assess whether there is evidence suggestive of a potential benefit in these patients with respect to endothelial function, cardiovascular outcomes, symptom control, QoL, and exercise capacity.

\section{Methods}

Studies were identified by conducting systematic searches of PubMed, EMBASE, and the Cochrane Library up until March 13, 2018. Searches included a mix of MeSH and free text terms related to syndrome X, microvascular angina, non-obstructive coronary artery disease, exercise training, cardiac rehabilitation, and endothelial function. Additionally, systematic reviews and reference lists of papers were hand searched for additional studies. All clinical trials with ET/CR in symptomatic patients with NOCAD were included. The selection was not limited to randomized controlled trials because of the paucity of data in this field. Articles written in languages other than English were excluded. 
Eight original articles included 218 patients, of which 162 underwent an intervention, and 56 served as controls. The majority of patients in these studies were women $(97.7 \%)$. Inclusion criteria were ongoing exertional chest pain (CP) without significant CAD on CT scan or coronary angiography, and the majority of studies required a positive exercise stress test or perfusion scan. The studies were conducted in five different countries (UK, Poland, Sweden, Brazil, Iran). Four of the studies were randomized, controlled clinical studies [12-15].

The first study on physical training in patients with CSX was published by Eriksson et al. in 2000 [13]. The authors randomized women into three groups which consisted of 8-week body-awareness (BA) program followed by 8 weeks of ET (total of 16 weeks), 8 weeks of ET alone, or a no-intervention control group [13]. Exercise training entailed cycle ergometer training three times a week for $30 \mathrm{~min}$ at $50 \%$ of peak work rate, which was determined at the beginning of the study. Endpoints were exercise capacity, urinary excretion of catecholamines and cortisol as an indicator for "negative stress," adenosine sensitivity test as a measure for pain sensitivity, and assessment of flowmediated dilation (FMD) as an indicator of endothelial function. The authors were able to show that ET but not BA improved the exercise capacity by $36 \%$ and oxygen uptake by $26 \%$. Time to pain onset changed significantly after ET, but not after sole BA training. Pain sensitivity was not affected by BA or ET. Cortisol levels decreased after BA, whereas catecholamine levels remained unchanged. There was a trend towards an improved endothelial function in the ET cohort [13].

The same study group examined 21 women in 2002 [12]. They randomized women into three groups (A, ET; B, Jacobson relaxation therapy, autogenous training; $\mathrm{C}$, control group). ET consisted of the same protocol as in the prior study [13]. QoL and coping capacity were examined by three different questionnaires (SOC, SCI-93, SIP) [12]. Health-related QoL improved in both the ET and the relaxation therapy groups, which was not associated with improved coping strategies [12].

In 2008, Asbury et al. published data on 64 women with diagnostic criteria for CSX, which were randomized into two groups [15]. One group underwent a standard 8-week group-based phase III CR program, which included an 80-min hospital-based circuit training once a week with the aim to exercise at $60-75 \%$ of their age-predicted heart rate reserve [15]. This was complemented by a home exercise session once a week. The other half had an 8-week symptom-monitoring program only. The patients' physical capacity was assessed by progressive shuttle walk (PSW) test. Each patient was asked to complete a symptom-monitoring diary daily and to fill out five different questionnaires to assess their psychological health (Health Anxiety Questionnaire, Hospital Anxiety and Depression Scale, SF-36, Cardiac Anxiety Questionnaire, Demographic Information Scale) at the beginning and at the end of the study. Compared to controls, women in the ET group showed significantly reduced symptom severity and psychological morbidity and were able to walk a longer distance in the PSW test [15].

Feizi et al. examined 40 women with CSX in Iran in 2012 [14]. Patients were randomized into four groups ((1) control, (2) CR only, (3) progressive muscle 
relaxation (PMR) only, (4) CR + PMR). The PMR group was instructed to perform Jacobson's method for 15 min per day at home. The CR group performed a home-based phase III program that consisted of an initial 25min walk, which gradually increased to $40 \mathrm{~min}$ at $60-65 \%$ of maximum heart rate, three times per week, for 8 weeks. The fourth group combined PMR and CR. Outcomes were assessed by the SF-36 questionnaire. In all three intervention groups, QoL improved. However, the combination of CR and PMR led to the most pronounced improvement of QoL scores [14].

Carvalho et al. published data from Brazil in 2014 [16]. Patients were included with exertional $\mathrm{CP}$, normal coronary angiography, and presence of $\geq 2$ myocardial segments with reversible perfusion defects (RPDs) identified by SPECT myocardial perfusion scan. This was the only study which included men (5/12). Patients underwent a 4-month rehabilitation program that consisted of three sessions per week of treadmill training at $60-85 \%$ of peak $\mathrm{VO}_{2}$. The program also included global stretching ( $5 \mathrm{~min})$, resistance exercise (10 $\mathrm{min})$, and relaxation ( $5 \mathrm{~min}$ ). At the end of the program, SPECT was repeated, as well as application of the SF-36 questionnaire. The authors showed a 14\% increase in oxygen uptake and were able to show a significant reduction in myocardial ischemia on SPECT (SDS, summed difference score). There was a significant improvement of the SF-36 scores in all categories but general health status [16]. In a further study, Carvalho et al. compared the same patient population with a healthy control group in regard to left ventricular ejection fraction (LVEF) at peak exercise on radionuclide ventriculography [17]. Compared to healthy controls, the LVEF in patients with MA did not rise significantly at peak exertion and this did not improve with exercise training over 4 months [17].

The latest studies were published by Szot et al. from Poland who examined 55 women with CSX and RPDs on SPECT [18]. Patients underwent an exercise program for 3 months with ET scheduled three times a week for 90 min (30min warm-up, 30-min bicycle ergometer, 30-min relaxation). The training intensity was increased from 70 to $100 \mathrm{~W}$ after 4 weeks and to $120 \mathrm{~W}$ after another month. The intensity was reduced if $80 \%$ of maximum heart rate was achieved. After 3 months, the Ferrans \& Powers Quality of Life Index questionnaire was repeated, as well as SPECT. The authors demonstrated a 20\% increase in exercise capacity, as well as an improved perfusion on SPECT during exercise. There was a weak correlation between improved SDS and QoL [18]. The same study group published another paper with the same cohort, showing an increase in exercise capacity, improved systolic and diastolic blood pressure, reduced body mass index (BMI), and improved SDS [19].

In summary, the duration of the exercise programs varied from 8 weeks to 4 months, with most of the programs lasting 8 weeks (Table 1). The majority of interventions were hospital-based. Only Feizi et al. prescribed a solely home-based program, whereas Asbury et al. used a mix of hospitaland home-based sessions. Most of the exercise programs performed three training sessions per week that lasted at least $30 \mathrm{~min}$, only Asbury et al. prescribed three sessions per week of longer duration (Table 1). The minimum training time per week was 90 min. The training mostly consisted of cycle ergometer but also included walking for the homebased program. Exercise intensity varied between the different studies but most authors tried to achieve a moderate intensity, guided by percentage of maximum heart rate $[14,18,19]$, heart rate reserve [15], peak $\mathrm{VO}_{2}$ [16], 


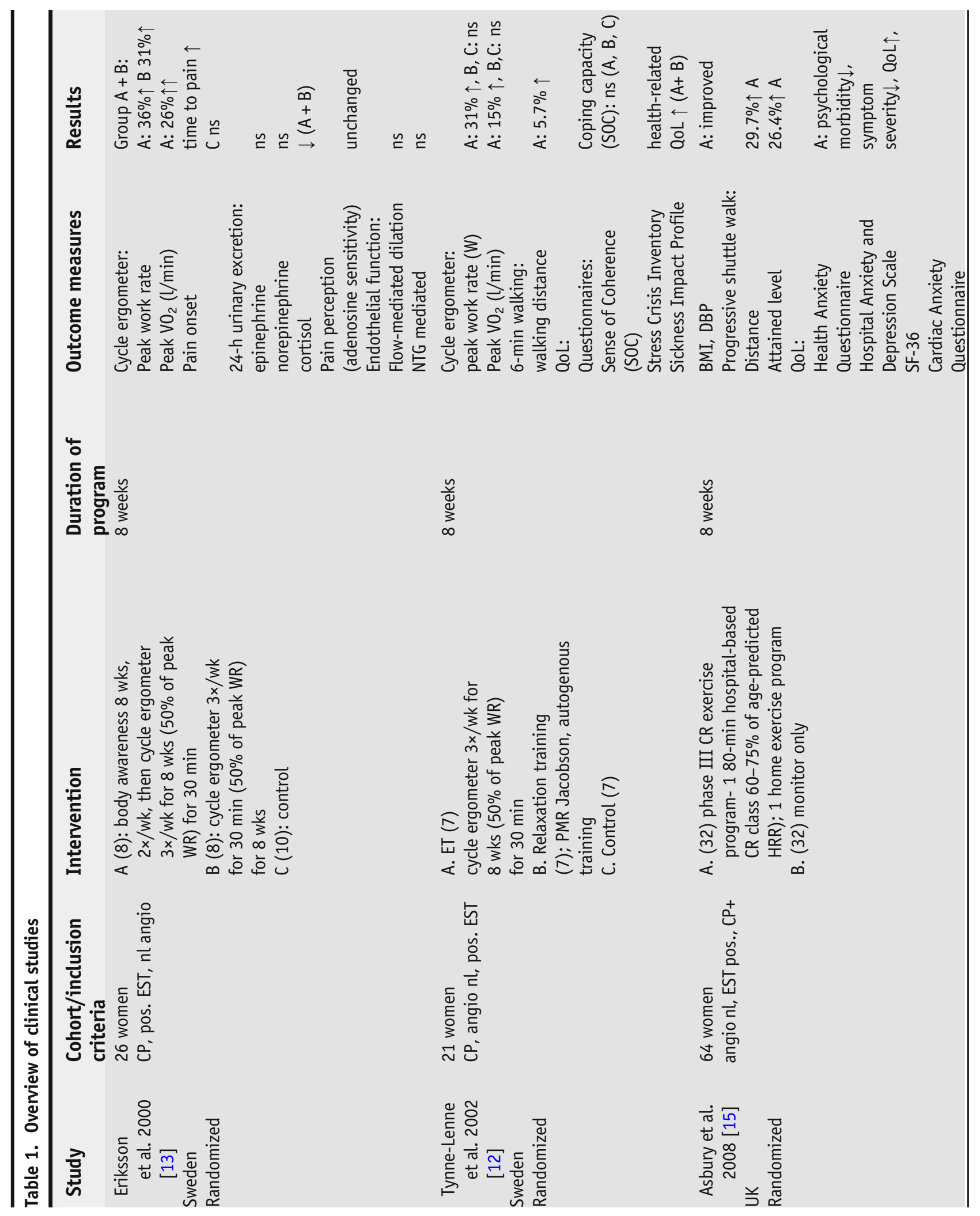



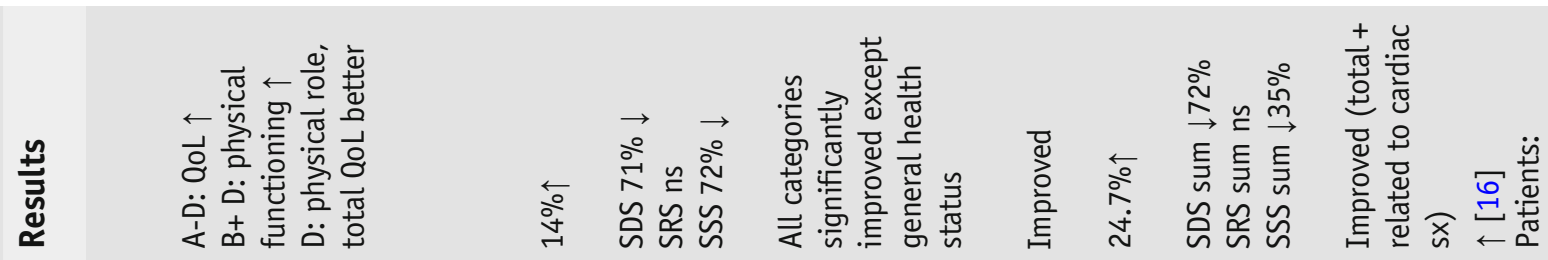

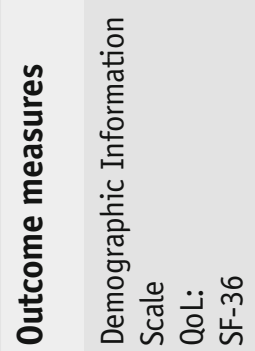
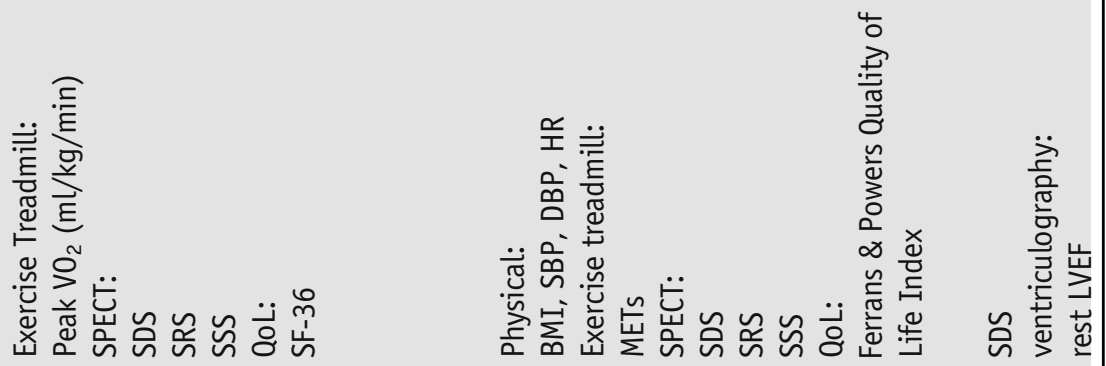

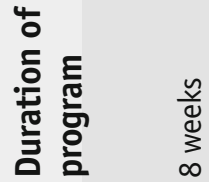

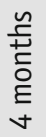

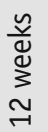
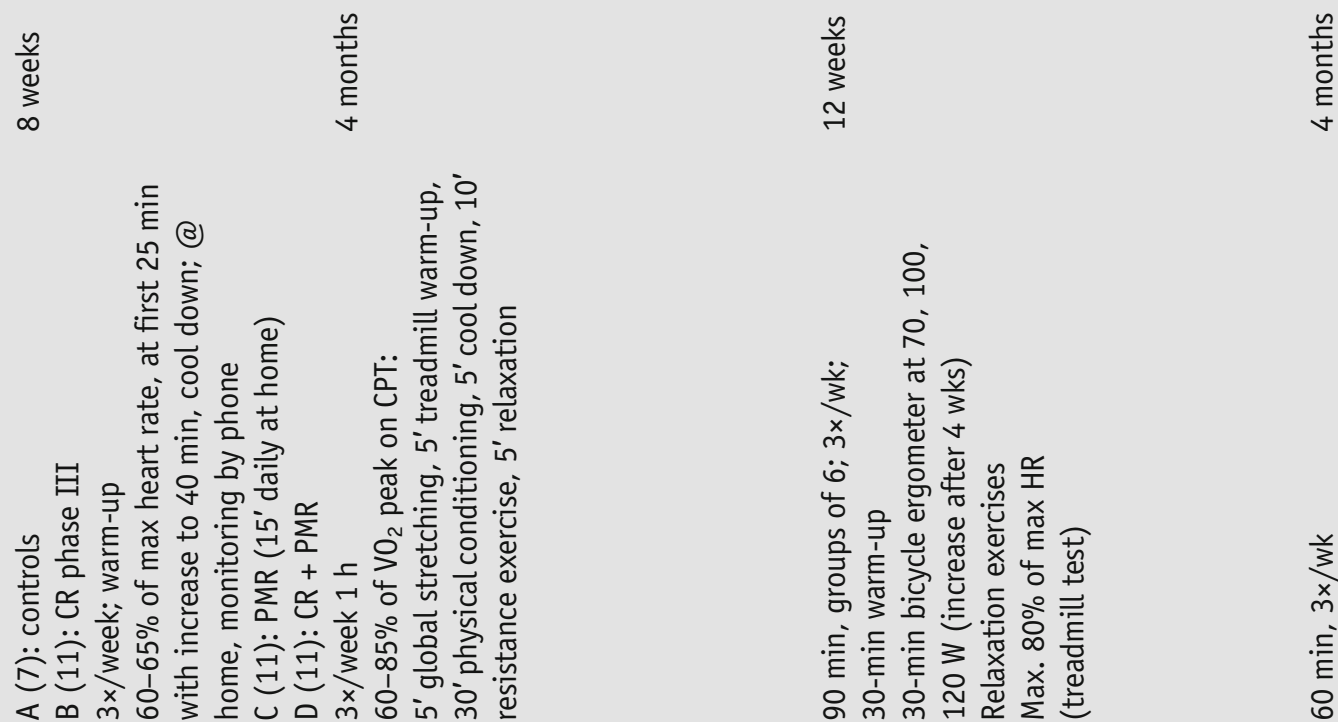

혼

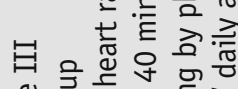
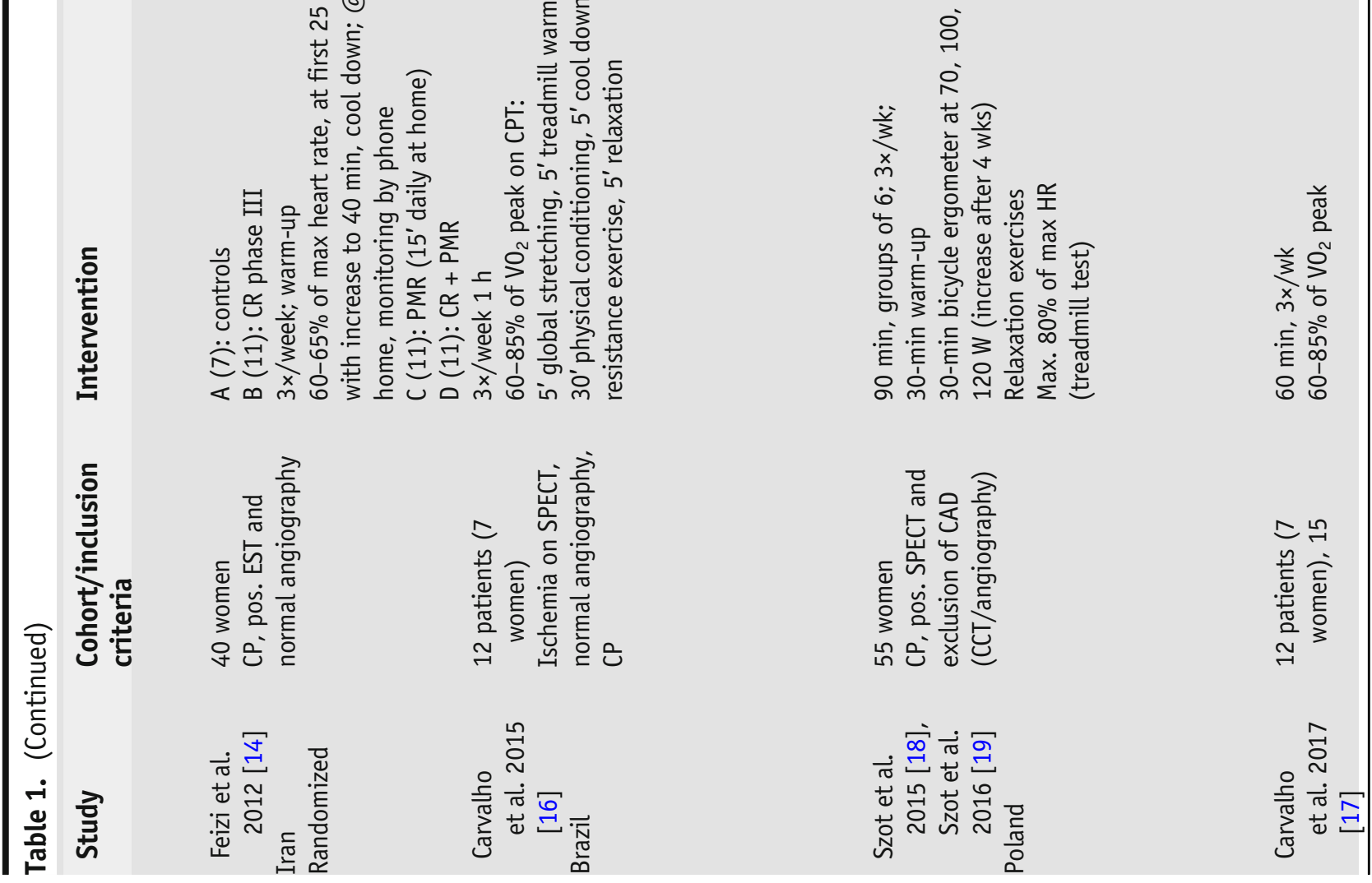


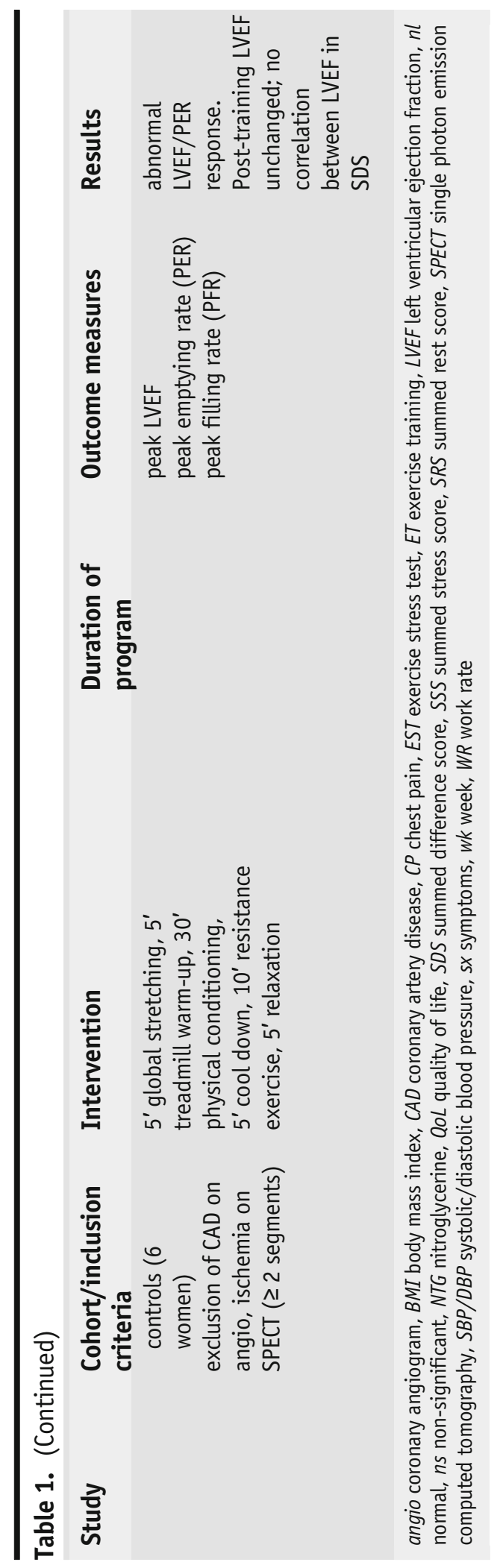




\section{Discussion}

or peak work rate $[12,13]$ (Table 1). Figure 1 summarizes the positive effects of CR in symptomatic patients with NOCAD. ET improved exercise capacity in all studies, albeit to a varying extent. In addition, ET improved QoL and symptom severity in most studies. The combination of relaxation therapies with ET or relaxation therapy alone appeared to have a positive impact on QoL parameters in three studies.

Review of the current literature on effects of physical exercise in NOCAD patients with angina yielded a limited number of studies. ET appears to be beneficial in patients with CSX/microvascular angina who are notoriously difficult to treat with one third remaining symptomatic in spite of treatment [5]. Albeit training protocols differed between the study populations, moderate activity 2-3 times per week (minimum of $90 \mathrm{~min} /$ week) appears to improve physical functioning over 8 weeks. This is of importance since impaired physical functioning is a feature of symptomatic patients with NOCAD [7]. Furthermore, Jespersen et al. showed that severity of persistent angina is associated with impaired physical functioning and QoL in NOCAD patients [7]. Asbury et al. demonstrated that ET reduced the severity of pain episodes, which may partially explain the improved QoL [15]. It remains uncertain whether this is mediated by an increased myocardial perfusion through improved coronary endothelial function. None of the studies looked at coronary endothelial function directly. One study showed a trend towards improvement of peripheral endothelial function using FMD, despite the fact that the study was underpowered [13]. There is also one case report which supports this hypothesis by showing a reversal of RPDs together with an improved peripheral endothelial function [20]. Furthermore, studies in diabetic patients with CAD showed that coronary endothelial function is improved by ET, which might explain the positive effects observed in the NOCAD population [21].

Although a variety of exercise protocols seem to have a beneficial effect on symptomatic patients with NOCAD, the optimal training strategy and guidance

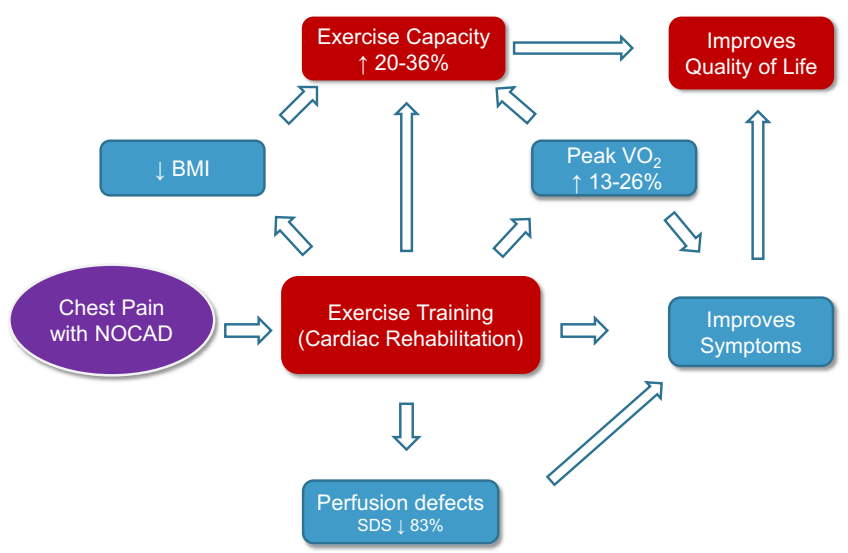

Fig. 1. Beneficial effects of cardiac rehabilitation in patients with NOCAD. BMI, body mass index; SDS, summation difference score; NOCAD, non-obstructive coronary artery disease, $\mathrm{VO}_{2}$, oxygen uptake. 
remain uncertain. Carvalho et al. used a longer duration of the CR program (4 months) but did not adapt the training intensity over the course of the program and patients had only a mild increase in $\mathrm{VO}_{2}$ of $13 \%$ [16]. The authors were not able to show an improvement in general health status as assessed by SF-36. This may be related to the limited training effect. Another possible explanation is that the SF-36 questionnaire is a well-established generic questionnaire, which evaluates parameters like general health perception, social functioning, and mental health [22]. Application of a more disease-specific questionnaire such as the Seattle Angina questionnaire might have yielded different results [23].

As recommended by guidelines, authors chose mostly a moderate training intensity, although guidance parameters varied between the studies. A metaanalysis recently showed that high-intensity training improves endothelial function in patients with cardiovascular disease [24], hereby raising the question whether it might be a time-efficient alternative or addition in NOCAD patients. Furthermore, training strategies like skiing appear to have a beneficial effect on endothelial health, which might broaden the recommended exercise spectrum, at least in alpine regions [25].

The reported trials have several limitations. Firstly, they were all small with low patient numbers in each treatment group, hereby limiting statistical power. In addition, not all of the studies were randomized. Secondly, the majority of studies included only women $(97.7 \%)$. Although cardiac syndrome $\mathrm{X}$ is more common in women, it is well established that it also occurs in men, with up to

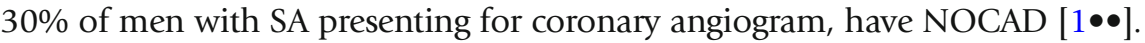
Given that the studies were limited to women, we can only speculate whether ET has the same positive effect in men.

Outcome measures in the reported trials consisted mostly of parameters for exercise capacity, easily measurable physical values, and QoL assessed by questionnaires. All outcomes were evaluated in the short term, directly after completion of the CR program. No data is available on the long-term effects of CR programs in NOCAD, and whether the beneficial effect is sustained over time. Furthermore, it would be interesting whether this transfers into hard endpoints like less frequent hospitalization, lower treatment costs, and possibly an improved outcome. For a long time, symptomatic patients with NOCAD were assured of the benign nature of their condition. However, recent data points towards an adverse outcome of these patients in regard to myocardial infarction, cardiovascular, and all-cause mortality $[1 \bullet \bullet, 26]$. Therefore, it would be intriguing to assess whether CR also leads to an improved cardiovascular outcome in this patient population.

Current studies on the effect of ET in symptomatic patients with NOCAD are promising but larger, randomized studies with inclusion of male patients are needed to evaluate the benefit of ET on hard endpoints and the long-term effect of ET. Furthermore, a study protocol should include randomized groups to determine the optimal training protocol in regard to training intensity, duration, and inclusion of relaxation techniques. Furthermore, it would be of interest to include vascular function studies to gain further insight into the pathophysiological mechanisms. 


\section{Conclusion}

Available evidence suggest that exercise training in women with angina and NOCAD has a beneficial effect on exercise capacity, severity of symptoms, and quality of life and appears to improve myocardial perfusion defects. Further, larger trials with inclusion of men are warranted in this challenging population, which is commonly subjected to numerous medical and invasive therapies of uncertain efficacy.

\section{Compliance with Ethical Standards}

\section{Conflict of Interest}

The authors declare that they have no conflicts of interest.

Human and Animal Rights and Informed Consent

This article does not contain any studies with human or animal subjects performed by any of the authors.

\section{Open Access}

This article is distributed under the terms of the Creative Commons Attribution 4.0 International License (http://creativecommons.org/licenses/by/4.0/), which permits unrestricted use, distribution, and reproduction in any medium, provided you give appropriate credit to the original author(s) and the source, provide a link to the Creative Commons license, and indicate if changes were made.

\section{References and Recommended Reading}

Papers of particular interest, published recently, have been highlighted as:

- Of importance

- Of major importance

1.• Jespersen L, Hvelplund A, Abildstrom SZ, Pedersen F, Galatius S, Madsen JK, et al. Stable angina pectoris with no obstructive coronary artery disease is associated with increased risks of major adverse cardiovascular events. Eur Heart J. 2012;33(6):734-44. https://doi. org/10.1093/eurheartj/ehr331.

A large, comtemporary study, which demonstrated a worse prognosis of symptomatic patients with NOCAD.

2. Shaw J, Anderson T. Coronary endothelial dysfunction in non-obstructive coronary artery disease: risk, pathogenesis, diagnosis and therapy. Vasc Med. 2016;21(2):146-55. https://doi.org/10.1177/ $1358863 X 15618268$.
Contempary review on the pathophysiology, diagnosis, and treatment of endothelial dysfunction in NOCAD.

3. Montalescot G, Sechtem U, Achenbach S, Andreotti F, Arden C, Budaj A, et al. 2013 ESC guidelines on the management of stable coronary artery disease: the Task Force on the management of stable coronary artery disease of the European Society of Cardiology. Eur Heart J. 2013;34(38):2949-3003. https://doi.org/10.1093/ eurheartj/eht296.

4. Shaw LJ, Merz CN, Pepine CJ, Reis SE, Bittner V, Kip KE, et al. The economic burden of angina in women with suspected ischemic heart disease: 
results from the National Institutes of Health-National Heart, Lung, and Blood Institute-sponsored Women's ischemia syndrome evaluation. Circulation. 2006;114(9):894-904.

5. Lamendola P, Lanza GA, Spinelli A, Sgueglia GA, Di Monaco A, Barone L, et al. Long-term prognosis of patients with cardiac syndrome X. Int J Cardiol. 2010;140(2):197-9. https://doi.org/10.1016/j.ijcard. 2008.11.026.

6. $\quad$ Ong P, Athanasiadis A, Sechtem U. Treatment of angina pectoris associated with coronary microvascular dysfunction. Cardiovasc Drugs Ther. 2016;30(4):351-6. https://doi.org/10.1007/ s10557-016-6676-z.

Current review on pharmacotherapy of microvascular angina.

7. Jespersen L, Abildstrom SZ, Hvelplund A, Prescott E. Persistent angina: highly prevalent and associated with long-term anxiety, depression, low physical functioning, and quality of life in stable angina pectoris. Clin Res Cardiol. 2013;102(8):571-81. https://doi.org/10.1007/s00392-013-0568-z.

8. Olson MB, Kelsey SF, Matthews K, Shaw LJ, Sharaf BL, Pohost GM, et al. Symptoms, myocardial ischaemia and quality of life in women: results from the NHLBIsponsored WISE study. Eur Heart J.

2003;24(16):1506-14.

9. Hambrecht R, Wolf A, Gielen S, Linke A, Hofer J, Erbs S, et al. Effect of exercise on coronary endothelial function in patients with coronary artery disease. N Engl J Med. 2000;342(7):454-60. https://doi.org/10.1056/ NEJM200002173420702.

10. Pearson MJ, Smart NA. Effect of exercise training on endothelial function in heart failure patients: a systematic review meta-analysis. Int J Cardiol. 2017;231:234-43. https://doi.org/10.1016/j.ijcard.2016.12.145.

11. Dalal HM, Doherty P, Taylor RS. Cardiac rehabilitation. BMJ. 2015;351:h5000. https://doi.org/10.1136/ bmj.h5000.

12. Tyni-Lenne R, Stryjan S, Eriksson B, Berglund M, Sylven C. Beneficial therapeutic effects of physical training and relaxation therapy in women with coronary syndrome X. Physiother Res Int. 2002;7(1):35-43.

13. Eriksson BE, Tyni-Lennè R, Svedenhag J, Hallin R, Jensen-Urstad K, Jensen-Urstad M, et al. Physical training in syndrome $\mathrm{X}$ : physical training counteracts deconditioning and pain in syndrome X. J Am Coll Cardiol. 2000;36(5):1619-25. https://doi.org/ 10.1016/S0735-1097(00)00931-1.

14. Feizi A, Ghaderi C, Dehghani MR, Khalkhali HR, Sheikhi S. Effect of phase III cardiac rehabilitation and relaxation on the quality of life in patients with cardiac syndrome X. Iran J Nurs Midwifery Res.

2012;17(7):547-52.

15. Asbury EA, Slattery C, Grant A, Evans L, Barbir M, Collins P. Cardiac rehabilitation for the treatment of women with chest pain and normal coronary arteries. Menopause. 2008;15(3):454-60. https://doi.org/10. 1097/gme.0b013e31815982eb.
16. Carvalho EE, Santi GL, Crescencio JC, de Oliveira LF, dos Reis DC, Figueiredo AB, et al. Pilot study testing the effect of physical training over the myocardial perfusion and quality of life in patients with primary microvascular angina. J Nucl Cardiol. 2015;22(1):130-7. https://doi.org/10. 1007/s12350-014-9949-6.

17. Carvalho EE, Crescencio JC, Santi GL, Oliveira LF, Schwartzmann PV, Gallo L, Jr. et al. Physical training improves myocardial perfusion but not left ventricular function response to exercise in patients with microvascular angina. Q J Nucl Med Mol Imaging 2017. doi:https://doi.org/10.23736/S1824-4785.17.029302.

18. Szot W, Zajac J, Kostkiewicz M, Owoc J, Bojar I. Cardiac rehabilitation: a good measure to improve quality of life in peri- and postmenopausal women with microvascular angina. Ann Agric Environ Med. 2015;22(2):390-5. https://doi.org/10.5604/12321966.1152100.

19. Szot W, Zajac J, Kubinyi A, Kostkiewicz M. The effects of cardiac rehabilitation on overall physical capacity and myocardial perfusion in women with microvascular angina. Kardiol Pol. 2016;74(5):431-8. https:// doi.org/10.5603/KP.a2015.0198.

20. Carvalho EE, Crescencio JC, Elias J Jr, Brito LB, Gallo L Jr, Simoes MV. Improved endothelial function and reversal of myocardial perfusion defects after aerobic physical training in a patient with microvascular myocardial ischemia. Am J Phys Med Rehabil. 2011;90(1):59-64. https://doi.org/10.1097/PHM. 0b013e3181ddd6bb.

21. Sixt S, Beer S, Bluher M, Korff N, Peschel T, Sonnabend $\mathrm{M}$, et al. Long- but not short-term multifactorial intervention with focus on exercise training improves coronary endothelial dysfunction in diabetes mellitus type 2 and coronary artery disease. Eur Heart J. 2010;31(1):112-9. https://doi.org/10.1093/eurheartj/ ehp398.

22. Brazier JE, Harper R, Jones NM, O'Cathain A, Thomas KJ, Usherwood T, et al. Validating the SF-36 health survey questionnaire: new outcome measure for primary care. BMJ. 1992;305(6846):160-4.

23. Spertus JA, Winder JA, Dewhurst TA, Deyo RA, Fihn SD. Monitoring the quality of life in patients with coronary artery disease. Am J Cardiol. 1994;74(12):1240-4.

24. Kolmos M, Krawcyk RS, Kruuse C. Effect of highintensity training on endothelial function in patients with cardiovascular and cerebrovascular disease: a systematic review. SAGE Open Med.

2016;4:2050312116682253. https://doi.org/10.1177/ 2050312116682253.

25. Niederseer D, Steidle-Kloc E, Mayr M, Muller EE, Cadamuro J, Patsch W, et al. Effects of a 12-week alpine skiing intervention on endothelial progenitor cells, peripheral arterial tone and endothelial biomarkers in the elderly. Int J Cardiol. 2016;214:343-7. https://doi.org/10.1016/j.ijcard. 2016.03.229. 
26. Sharaf B, Wood T, Shaw L, Johnson BD, Kelsey S, Anderson $\mathrm{RD}$, et al. Adverse outcomes among women presenting with signs and symptoms of ischemia and no obstructive : findings from the National Heart, Lung, and
Blood Institute-sponsored Women's Ischemia Syndrome Evaluation (WISE) angiographic core laboratory. Am Heart J. 2013;166(1):134-41. https://doi.org/10.1016/j. ahj.2013.04.002. 